\title{
Endothelial nitric oxide synthase enhancer for protection of endothelial function from asymmetric dimethylarginine-induced injury in human internal thoracic artery
}

\author{
Chao Xuan, MPhil, ${ }^{\mathrm{a}}$ Feng-Jun Chang, MD, ${ }^{\mathrm{b}}$ Xiao-Cheng Liu, MD, ${ }^{\mathrm{a}}$ Xiao-Yan Bai, MD, ${ }^{\mathrm{a}}$ \\ Xiao-Long Liao, MD, ${ }^{\mathrm{b}}$ Guo-Wei He, MD, PhD, DSc, ${ }^{\text {a,c }}$ and Jing-Song Ou, MD, PhD, FCCP
}

\begin{abstract}
Objectives: Endogenous nitric oxide synthase inhibitor asymmetric dimethylarginine is a cardiovascular risk factor that is elevated in patients with coronary artery disease. We hypothesized that novel endothelial nitric oxide synthase enhancer AVE3085 might improve the endothelial function altered by asymmetric dimethylarginine in the human internal thoracic artery.
\end{abstract}

\begin{abstract}
Methods: Cumulative concentration-relaxation curves to acetylcholine ( -11 to $-5 \log \mathrm{mol} / \mathrm{L})$ were established in left internal thoracic artery rings $(n=65)$ from 27 patients undergoing coronary artery bypass grafting in precontraction induced by $\mathrm{U} 46619(-8 \mathrm{log} \mathrm{mol} / \mathrm{L})$ in the absence or presence of asymmetric dimethylarginine $(100 \mu \mathrm{mol} / \mathrm{L})$ or AVE3085 $(30 \mu \mathrm{mol} / \mathrm{L})$. Protein expressions of endothelial nitric oxide synthase and levels of superoxide anion production were detected.
\end{abstract}

Results: Maximal relaxation induced by acetylcholine was significantly attenuated by asymmetric dimethylarginine $(12.7 \% \pm 2.3 \%$ vs $35.3 \% \pm 5.0 \%$ in control; $P<.05)$ and significantly restored by AVE3085 $(23.4 \% \pm 2.8 \%$; $P<.05)$. AVE3085 also markedly restored endothelial nitric oxide synthase expression $(0.29 \pm 0.008 ; P=.012)$ reduced by asymmetric dimethylarginine $(0.05 \pm 0.04$ vs $0.36 \pm 0.03$ in control; $P=.014)$. Increased superoxide anion production by asymmetric dimethylarginine $(2.97 \pm 0.25$ vs $0.51 \pm 0.10$ relative light units/[s/mg] in control; $P<.05)$ was inhibited by AVE3805 $(0.62 \pm 0.104$ relative light units/[s/mg]; $P<.05)$.

Conclusions: AVE3085 may restore endothelium-dependent relaxation reduced by asymmetric dimethylarginine through upregulation of endothelial nitric oxide synthase expression and inhibition of production of superoxide anion in human internal thoracic artery. These findings provide new insights into endothelial protection of coronary bypass grafting vessels to improve long-term patency of grafts. ( $\mathrm{J}$ Thorac Cardiovasc Surg 2012;144:697-703)

Asymmetric dimethylarginine (ADMA) is produced in human cells during proteolysis of methylated nuclear proteins.

\footnotetext{
From TEDA International Cardiovascular Hospital, ${ }^{\mathrm{a}}$ Medical College, Nankai University, Tianjin, China; the Division of Cardiac Surgery, ${ }^{\mathrm{b}}$ The Key Laboratory of Assisted Circulation, Ministry of Health, The First Affiliated Hospital, Sun Yatsen University Guangzhou, China; and the Department of Surgery, ${ }^{\mathrm{c}}$ Oregon Health and Science University, Portland, Ore.

Fully supported by grants from the National Natural Science Foundation of China (No. 81170148), International S \& T Cooperation Program of China Technology (2009DFB30560), National Basic Research Program of China (2010CB529500), and Tianjin Municipal Science and Technology Commission (09ZCZDSF04200 \& 10JCYBJC26400) (to G-W.H.); the National Natural Science Foundation of China (No. 30971261) and The Fundamental Research Funds for the Central Universities, Ministry of Education of China (to J-S.O.); and Guangdong Natural Science Fund Committee (No. 9251008901000003) and Bureau of Science and Information Technology of Guangzhou (2010GN-E00221).

Disclosures: Authors have nothing to disclose with regard to commercial support.

C.X. and F-J.C. share the position of first author.

Received for publication Sept 20, 2011; revisions received Dec 1, 2011; accepted for publication Jan 4, 2012; available ahead of print Feb 16, 2012.

Addresses for reprints: Guo-Wei He, MD, PhD, DSc, TEDA International Cardiovascular Hospital, Medical College, Nankai University, No. 61, The 3rd, TEDA, Tianjin, 300457, China (E-mail: heguowei@ nankai.edu.cn or gwhezj@hotmail. com). or Jing-song Ou, MD, PhD, FCCP, Division of Cardiac Surgery, The First Affiliated Hospital, Sun Yat-sen University, 58 Zhong Shan Er Rd, Guangzhou, 510080, China. (E-mail: oujs@mail.sysu.edu.cn or oujs2000@yahoo.com). $0022-5223 / \$ 36.00$

Copyright (c) 2012 by The American Association for Thoracic Surgery doi:10.1016/j.jtcvs.2012.01.020
}

It acts as an endogenous inhibitor of endothelial nitric oxide synthase (eNOS) by competing with L-arginine, and this in turn causes endothelial dysfunction. ${ }^{1}$ Alternatively, ADMA may uncouple eNOS, leading not only to the loss of nitric oxide but also to increased superoxide production in the vascular endothelium and induced apoptosis of endothelial cells. ${ }^{2}$ Superoxide anion generation also can scavenge nitric oxide to impair vascular function and inhibit vasodilation. ${ }^{3}$ The occurrence of cardiovascular end points in high-risk patients has been found to be directly and independently associated with elevated ADMA concentrations in patients with coronary artery disease, ${ }^{4}$ diabetes mellitus, ${ }^{5}$ and chronic heart failure. ${ }^{6}$

Recently, 2 new small-molecular weight compounds (AVE9448 and AVE3085) were developed to increase endothelial nitric oxide production by simultaneous upregulation of eNOS expression and reversal of eNOS uncoupling. ${ }^{7}$ It has been demonstrated that impaired eNOS production is enhanced by AVE3085 treatment in the hind limb of severely diabetic animals and that treatment with AVE3085 significantly increases eNOS messenger RNA and protein levels in cardiac tissue. ${ }^{8,9}$ Further, we have recently demonstrated that use of AVE3085 protects porcine coronary endothelium from ischemia-reperfusion or hypoxia-reoxygenation injury and 


$$
\begin{aligned}
& \text { Abbreviations and Acronyms } \\
& \text { ADMA }=\text { asymmetric dimethylarginine } \\
& \text { ANOVA }=\text { analysis of variance } \\
& \text { CI }=\text { confidence interval } \\
& \text { DMSO }=\text { dimethyl sulfoxide } \\
& \mathrm{E}_{\max }=\text { maximal relaxation } \\
& \mathrm{EC}_{50}=50 \% \text { effective concentration } \\
& \text { eNOS }=\text { endothelial nitric oxide synthase } \\
& \text { LITA }=\text { left internal thoracic artery } \\
& \text { RLU }
\end{aligned}
$$

restores impaired endothelial function in a hypertensive model. $^{10,11}$ In addition, AVE3085 has been shown to ameliorate endothelial dysfunction in $\mathrm{db} / \mathrm{db}$ mice through increased nitric oxide bioavailability, which reduces oxidative stress in the vascular wall. ${ }^{12}$ This study was therefore designed to test the hypothesis that ADMA has detrimental effects on the endothelial function in the human internal thoracic artery, including both decreased nitric oxidemediated relaxation and increased superoxide anion production, and that AVE3085 could restore these functions.

\section{MATERIALS AND METHODS \\ Vessel Preparation}

The discarded left internal thoracic artery (LITA) segments from patients undergoing coronary artery bypass grafting $(n=27$; Table 1$)$ were immediately collected for transfer to the laboratory. Approval to use the discarded human vessel tissue was given by the ethics committee (institutional review board) of TEDA International Cardiovascular Hospital, Tianjin, China. During coronary artery bypass grafting with LITA grafts, the LITA was harvested as a full pedicle. The length required for grafting was carefully measured, usually immediately after the dissection of the LITA pedicle. The redundant distal end was trimmed off and immediately placed in

TABLE 1. Demographic and clinical characteristics of the patients $(\mathbf{n}=\mathbf{2 7})$

\begin{tabular}{lc}
\hline Age (y, mean \pm SEM) & $60.6 \pm 5.1$ \\
Sex (no.) & $11(40.7 \%)$ \\
$\quad$ Female & $16(59.3 \%)$ \\
$\quad$ Male & $13(48.1 \%)$ \\
Smoking habit (no.) & $26.11 \pm 3.40$ \\
Body mass index (kg/m ${ }^{2}$, mean \pm SEM) & \\
Blood pressure (mm Hg, mean \pm SEM) & $132.7 \pm 16.7$ \\
$\quad$ Systolic & $77.2 \pm 11.9$ \\
$\quad$ Diastolic & $19(70.4 \%)$ \\
Diabetes mellitus (no.) & $22(81.5 \%)$ \\
Unstable angina (no.) & $4.72 \pm 1.03$ \\
Total cholesterol (mmol/L, mean \pm SEM) & $1.74 \pm 0.95$ \\
Triglycerides (mmol/L, mean \pm SEM) & $1.08 \pm 0.25$ \\
HDL cholesterol (mmol/L, mean \pm SEM) & $3.05 \pm 0.86$ \\
LDL cholesterol (mmol/L, mean \pm SEM) & $27(100 \%)$ \\
Nitroglycerin* (no.) & $1(3.7 \%)$ \\
Dopamine* (no.) & \\
\hline$H D L$, High-density lipoprotein; $L D L$, low-density lipoprotein. $*$ Use of vasodilators or \\
vasoconstrictors during the harvesting of the left internal thoracic artery.
\end{tabular}

a container with oxygenated physiologic salt solution, maintained at $4^{\circ} \mathrm{C}$, and transferred to the laboratory within 5 to 10 minutes. The adherent connective tissue was carefully dissected, and the LITA was cut precisely into 3-mm long segments. Organ baths in the myograph were filled with Krebs solution, maintained at $37^{\circ} \mathrm{C}$ and aerated continuously with a mixture of $95 \%$ oxygen and $5 \%$ carbon dioxide. The modified Krebs solution had the following composition: sodium ion, $144 \mathrm{mmol} / \mathrm{L}$; potassium ion, $5.9 \mathrm{mmol} / \mathrm{L}$; calcium ion, $2.5 \mathrm{mmol} / \mathrm{L}$; magnesium ion, $1.2 \mathrm{mmol} / \mathrm{L}$; chloride ion, $128.7 \mathrm{mmol} / \mathrm{L}$; hydrogen carbonate ion, $25 \mathrm{mmol} / \mathrm{L}$; sulfate ion, 1.2 $\mathrm{mmol} / \mathrm{L}$; dihydrogen phosphate ion, $1.2 \mathrm{mmol} / \mathrm{L}$; and glucose, $11 \mathrm{mmol} / \mathrm{L}$.

\section{Myograph Techniques}

Human LITA ring segments were suspended on wire hooks in a 6-mL bath on a myograph modified for large vessel studies (model 610M; DMT Company, Aarhus, Denmark). Each ring segment resting unstretched on the wire hooks was equilibrated in Krebs solution for at least 60 minutes.

\section{Normalization}

A technique described previously in detail elsewhere was used in this study. ${ }^{13}$ Briefly, each ring segment was stretched up in progressive steps every minute to determine the individual length-tension curve. A computer iterative fitting program (Myodaq and Myodata version 2.01; Maastricht University, Maastricht, The Netherlands) was used to determine the exponential curve pressure and the internal diameter. At the end of each step, the internal diameter (in micrometers) and the corresponding wall tension (in millinewtons per square millimeter) were recorded. When the transmural pressure on the rings reached $100 \mathrm{~mm} \mathrm{Hg}$, as determined according to the length-tension curves, the stretching procedure was stopped, and the rings were released to $90 \%$ of their internal circumference at $100 \mathrm{~mm} \mathrm{Hg}^{13,14}$ This degree of passive tension was then maintained throughout the experiment.

\section{Western Blot Analysis of eNOS}

LITA samples were homogenized in lysis buffer (KeyGEN, Inc, Nanjing, China), and the lysates were incubated in ice for 1 hour followed by 10 minutes of centrifugation at $10,000 \mathrm{rpm}$. After the sample was heated at $100^{\circ} \mathrm{C}$ for 5 minutes to denature it, $120 \mu \mathrm{g}$ protein for each sample was separated by $8 \%$ polyacrylamide gel electrophoresis (Page Gel, Inc, San Diego, Calif) together with the prestained protein ladder (MBI Fermentas, Inc, Glen Burnie, Md). The proteins were transferred electrophoretically to the polyvinylidene fluoride membrane (Millipore, Billerica, Mass). The membrane was blocked with blocking buffer (tris-buffered saline solution, $0.1 \%$ polysorbate $20,5 \%$ nonfat dry milk) for 3 hours at room temperature and incubated with primary antibody against eNOS (1:1,000; Cell Signaling Technology, Inc, Boulder, Colo) overnight at $4^{\circ} \mathrm{C}$. Equivalent protein on the same lane was confirmed by stripping and reblotting with glyceraldehyde 3-phosphate dehydrogenase (1:1000; Cell Signaling Technology). The secondary goat antirabbit antibody conjugated to horseradish peroxidase (Santa Cruz Biotechnology, Inc, Santa Cruz, Calif) at a dilution of 1:5000 was added the next day. Finally, blots were developed with an enhanced chemiluminescence detection system (Amersham Pharmacia ECL reagents; GE Healthcare Biosciences, Piscataway, NJ) and exposed to X-ray films. The protein bands were quantified with QuantityOne software (Bio-Rad Laboratories, Inc, Hercules, Calif), normalized by glyceraldehyde 3-phosphate dehydrogenase, and expressed as multiples of control.

\section{Superoxide Anion Measurement in Human Thoracic Artery}

LITA vessels were gently cut open longitudinally to expose the endothelial surface and incubated in oxygenated Krebs buffer with drugs at $37^{\circ} \mathrm{C}$ for 2 hours before measurement. The segments were then rinsed briefly in Krebs-4-(2-hydroxyethyl)-1-piperazineethanesulfonic acid and maintained at $37^{\circ} \mathrm{C}$ for 30 minutes. Lucigenin-enhanced chemiluminescence 
was measured in each segment with low-concentration lucigenin $(5 \mu \mathrm{mol} /$ L). Time-based reading of the fluorescence microplate reader was recorded by softMax pro v5.0.1 software. The luminescence in relative light units (RLU) per second for each sample was averaged between 5 and 10 minutes. Values of blank chambers that contained the same reagents were recorded as background, and the background values were subtracted from those of the corresponding vessel samples. Data were normalized to vessel dry weight and were represented as RLU per second per milligram dry weight.

\section{Experimental Protocol}

Ring segments were precontracted with U46619 at a concentration of $10^{-8} \mathrm{~mol} / \mathrm{L}$. Our previous studies showed that U46619 at $10^{-8} \mathrm{~mol} / \mathrm{L}$ induced $60 \%$ to $80 \%$ of maximal contractile responses in the LITA ring segments. ${ }^{13,14}$

\section{Effects of ADMA and AVE3085 on Acetylcholine- Induced Endothelium-Dependent Relaxation}

The LITA ring segments from the same patient were allocated randomly into 3 groups ( $\mathrm{n}=8$ per group). In 1 group, the LITA ring segments were incubated with Krebs solution as a control. In the other 2 groups, the LITA ring segments were incubated with Krebs solution in the presence of ADMA (100 $\mu \mathrm{mo} / \mathrm{L})$ or ADMA (100 $\mu \mathrm{mol} / \mathrm{L})$ with AVE3085 $(30 \mu \mathrm{mol} / \mathrm{L}$, dissolved in dimethyl sulfoxide [DMSO]) for 1 hour before the precontraction was started.

The acetylcholine-induced relaxation in the presence of DMSO (the solvent of AVE3085) was also tested to exclude the effect of the solvent $(n=8$ in each group). In 1 group, the LITA segment was incubated with Krebs solution in the presence of ADMA ( $100 \mu \mathrm{mol} / \mathrm{L})$, and in the other group, the LITA segments were incubated with Krebs solution in the presence of ADMA $(100 \mu \mathrm{mol} / \mathrm{L})$ plus DMSO for 1 hour before the precontraction was started. The results were then compared with those of the Krebs solution control.

In separate rings $(n=4)$, the endothelium was mechanically removed by gently rubbing the luminal surface of the ring with small plastic tubing (endothelium-denuded vessels).

Ring segments were precontracted with $\mathrm{U} 46619\left(10^{-8} \mathrm{~mol} / \mathrm{L}\right)$. When the contraction reached a stable plateau, acetylcholine $\left(10^{-11}\right.$ to $\left.10^{-5} \mathrm{~mol} / \mathrm{L}\right)$ was applied to a cumulative concentration at an interval between doses to allow the relaxation induced by the previous dose to reach a plateau. The relaxation was expressed as percentage reversal of the U46619induced precontraction.

\section{Western Blot Analysis of eNOS}

Immediately after the previously described relaxation study, the LITA segments for control, ADMA, and ADMA with AVE3085 were flashfrozen in liquid nitrogen and stored at $-80^{\circ} \mathrm{C}$ for protein study $(\mathrm{n}=8$ in each group). The previously mentioned steps for eNOS measurement were then repeated. The total exposure time for the vessel to study of the protein expression was $5.56 \pm 0.25$ hours

\section{Measurement of Superoxide Anion}

The LITA ring segments from the same patient were allocated randomly into 3 groups ( $n=7$ in each group). After 30 minutes of equilibration of each segment in the organ chamber, the LITA ring segments were incubated with Krebs solution (control) or with Krebs solution in the presence of ADMA $(100 \mu \mathrm{mol} / \mathrm{L})$ or ADMA $(100 \mu \mathrm{mol} / \mathrm{L})$ with AVE3085 $(30 \mu \mathrm{mol} / \mathrm{L})$ for 2 hours. The previously mentioned steps for superoxide anion measurement were then repeated.

\section{Data Analysis}

Relaxation was expressed as the percentage decrease in isometric force induced by $\mathrm{U} 46619$. Mean maximal relaxation $\left(\mathrm{E}_{\max }\right)$ for each group was calculated from the maximal relaxation of different rings induced by acetylcholine. The effective concentration of acetylcholine that caused $50 \%$ of $\mathrm{E}_{\max }$ was defined as $\mathrm{EC}_{50}$. The $\mathrm{EC}_{50}$ was determined from each concentration-relaxation curve by a logistic, curve-fitting equation: $\mathrm{E}=\mathrm{MA}^{\mathrm{P}} /\left(\mathrm{A}^{\mathrm{P}}+\mathrm{K}^{\mathrm{P}}\right)$, where $E$ is response, $M$ is maximal relaxation $\left(\mathrm{E}_{\max }\right), A$ is concentration, $K$ is $\mathrm{EC}_{50}$, and $P$ is the slope parameter. From this fitted equation, the mean $\mathrm{EC}_{50} \pm \mathrm{SEM}$ was calculated for each group.

All statistical analyses were performed with the SPSS statistical software package (version 10.0; International Business Machines Corp, Armonk, NY). All values are expressed as mean \pm SEM. Statistical comparisons of the percentage relaxation under different treatments were performed by 2-way analysis of variance (ANOVA; general linear model) with repeated measures, followed by post hoc Bonferroni test to detect the individual differences. The comparison of the $\mathrm{E}_{\max }$ was performed with unpaired $t$ test. $\mathrm{EC}_{50}$, eNOS expression, and superoxide anion were compared by 1 -way ANOVA followed by post hoc Bonferroni test. The $95 \%$ confidence interval (CI) for difference was also shown when possible; $n$ values refer to number of ring segments from separate patients.

\section{Drugs}

Acetylcholine and ADMA were purchased from Sigma-Aldrich (St Louis, Mo). U46619 was from Cayman Chemical (Ann Arbor, Mich). AVE3085 was kindly provided by Sanofi-Aventis Deutschland GmbH (Frankfurt, Germany) and dissolved in DMSO. Others were dissolved in distilled water. Stock solutions of U46619 and acetylcholine were kept at $-20^{\circ} \mathrm{C}$ until required.

\section{RESULTS}

Among the groups of the patients whose LITAs were studied, there were no differences with regard to the clinical characteristics $(P>.05$ among the groups, details not shown; Table 1).

\section{Resting Parameters}

The internal diameter of the vessels at an equivalent transmural pressure of $100 \mathrm{~mm} \mathrm{Hg}$ was $1.82 \pm 0.07 \mathrm{~mm}$ $(\mathrm{n}=44)$, as determined from the computerized normalization procedure. The resting transmural pressure was $81.8 \pm$ $1.0 \mathrm{~mm} \mathrm{Hg}$ at $90 \%$ of this value. The resting force of the LITA rings was $2.3 \pm 0.09 \mathrm{~g}$.

\section{Effect of ADMA and AVE3085 on Acetylcholine- Induced Relaxation}

The acetylcholine-mediated relaxation was abolished in the endothelium-denuded rings (Figure 1, A). This result shows that the acetylcholine-induced relaxation in our experiments was endothelium dependent (Figure 1,B).

The acetylcholine-mediated relaxation was significantly inhibited in the presence of ADMA $(P<0.001$ by 2 -way ANOVA). In comparison with ADMA alone, addition of AVE3085 to ADMA significantly increased the acetylcholineinduced relaxation $(P=.001$ by 2 -way ANOVA; Figure $1, B)$.

Further, the $\mathrm{E}_{\max }$ induced by acetylcholine $(35.3 \% \pm$ $5.0 \%$ in control; $95 \%$ CI $25.1 \%-45.5 \%$ ) was significantly reduced in the rings treated with ADMA $(12.7 \% \pm 2.3 \%$; $95 \%$ CI $8.0 \%-17.3 \% ; P<.05$ by unpaired $t$ test $)$ or ADMA with AVE3085 (23.4\% $\pm 2.8 \%$; 95\% CI $17.6 \%-$ $29.1 \% ; P<.05$ by unpaired $t$ test). In addition, in comparison 


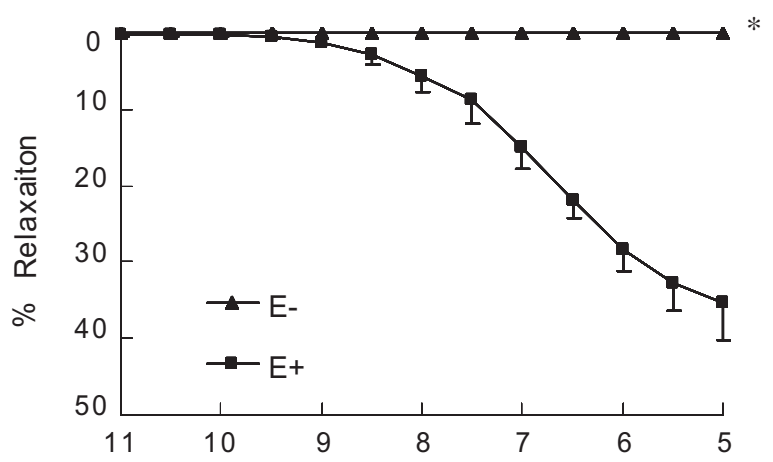

A

ACh $[-\log M]$

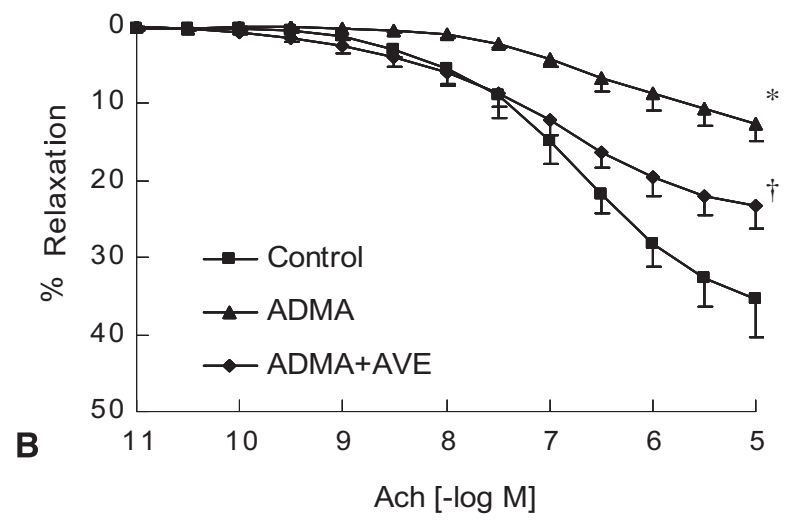

FIGURE 1. A, Mean concentration ( $\log \mathrm{M}$ )-relaxation (percentage reversal of U46619-induced contraction) curves for acetylcholine ( $A C h$ ) of left internal thoracic artery rings with endothelium $(E+, \mathrm{n}=8)$ or without endothelium $(E-, \mathrm{n}=4)$. B, Mean concentration-relaxation curves for acetylcholine (ACh) of left internal thoracic artery rings ( $\mathrm{n}=8$ in each group) in the absence or presence of asymmetric dimethylarginine (ADMA) or asymmetric dimethylarginine with AVE3085 ( $A D M A+A V E)$. Rings with endothelium were regarded as control. Values are expressed as mean \pm SEM. Asterisk indicates $P<.001$ versus control group; dagger indicates $P<.05$ versus asymmetric dimethylarginine group; 2 -way analysis of variance.

with ADMA alone, addition of AVE3085 to ADMA significantly increased the $\mathrm{E}_{\max }$ induced by acetylcholine $(P<.05$ by unpaired $t$-test).

With respect to the $\mathrm{EC}_{50}$, it was not significantly different among groups (control, $-6.99 \pm 0.30 \log \mathrm{mol} / \mathrm{L}$; ADMA, $-6.49 \pm 0.21 \log \mathrm{mol} / \mathrm{L} ;$ ADMA with AVE3085, $-7.27 \pm$ $0.33 \log \mathrm{mol} / \mathrm{L} ; P>.05$ by 1 -way ANOVA).

\section{Effect of DMSO on Acetylcholine-Induced Relaxation}

The acetylcholine-mediated relaxation was significantly inhabited in the presence of ADMA and ADMA with DMSO $(P<.001$ by 2 -way ANOVA) but was not significantly different between ADMA and ADMA with DMSO $(P>.05$ by 2 -way ANOVA; Figure 2$)$.

In comparison with ADMA alone $(14.9 \% \pm 1.2 \% ; 95 \%$ CI $12.4 \%-17.4 \%$ ), addition of DMSO to ADMA did not alter the $\mathrm{E}_{\max }$ induced by acetylcholine $(14.4 \% \pm 1.5 \%$, $95 \%$ CI $11.3 \%-17.5 \% ; P>.05$ by unpaired $t$ test).

The $\mathrm{EC}_{50}$ was not significantly different between the ADMA and ADMA with DMSO groups (ADMA, - -7.04 \pm $0.30 \log \mathrm{mol} / \mathrm{L} ;$ ADMA with DMSO, $-6.57 \pm 0.44 \log$ $\mathrm{mol} / \mathrm{L} ; P>.05$ by 1 -way ANOVA).

\section{Protein Expressions of eNOS: Effects of ADMA and AVE3085}

Expression of eNOS protein in LITA segments was significantly decreased after incubation with ADMA $(0.05 \pm$ 0.04 vs $0.36 \pm 0.03$ in control; $P=.014$ by 1 -way ANOVA). Addition of AVE3085 to ADMA markedly increased the expression level of eNOS $(0.29 \pm 0.008 ; P=.012$ vs ADMA alone by 1-way ANOVA), although there was still a difference between ADMA with AVE3085 and control $(0.29 \pm 0.008$ vs $0.36 \pm 0.03$ in control; $P=.026$ by 1-way ANOVA; Figure 3).

\section{Superoxide Anion Production: Effects of ADMA and AVE3085}

As shown in Figure 4, superoxide anion generation in LITA segments was low under basal conditions $(0.51 \pm$ $0.102 \mathrm{RLU} /[\mathrm{s} / \mathrm{mg}])$. ADMA dramatically increased superoxide anion production $(2.97 \pm 0.25 \mathrm{RLU} /[\mathrm{s} / \mathrm{mg}] ; P<.05$ vs control by 1-way ANOVA). Addition of AVE3085 significantly inhibited the ADMA-induced superoxide anion generation $(0.62 \pm 0.104 \mathrm{RLU} /[\mathrm{s} / \mathrm{mg}] ; P<.05$ vs ADMA group by 1-way ANOVA).

\section{DISCUSSION}

This study has demonstrated in human internal thoracic artery samples that (1) increased ADMA levels directly

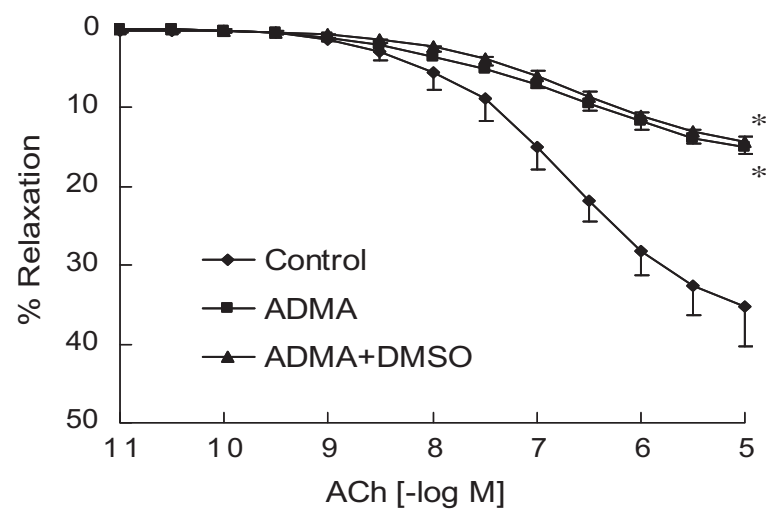

FIGURE 2. Mean concentration ( $\log M$ )-relaxation (percentage reversal of U46619-induced contraction) curves for acetylcholine (ACh) in the left internal thoracic artery rings ( $\mathrm{n}=8$ in each group) in the absence or presence of asymmetric dimethylarginine (ADMA) or asymmetric dimethylarginine with dimethyl sulfoxide $(A D M A+D M S O)$. Data are shown as mean \pm SEM. Asterisk indicates $P<.001$ versus control group by 2 -way analysis of variance. 


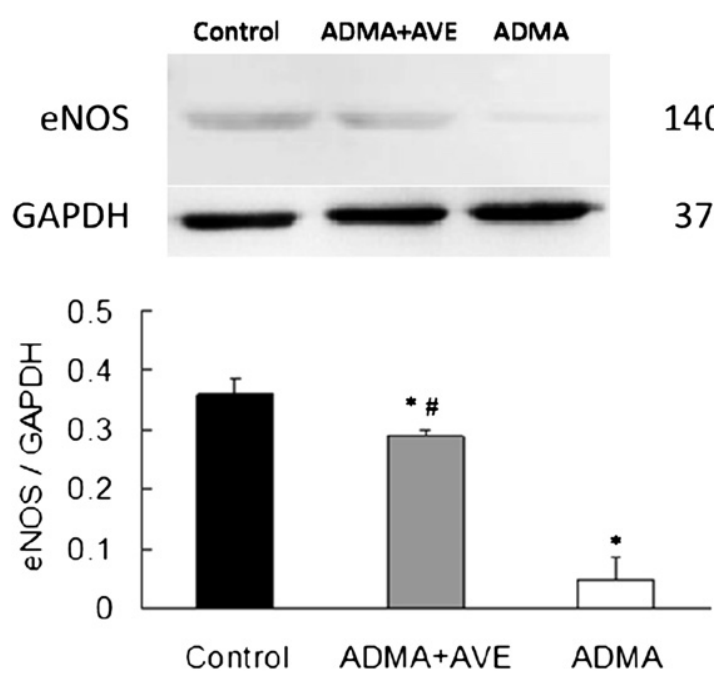

FIGURE 3. Protein expression of endothelial nitric oxide synthase $(e N O S)$ in human left internal thoracic artery rings. Arterial rings were incubated with asymmetric dimethylarginine ( $A D M A)$ in Krebs solution with or without the addition of AVE3085 (AVE) or for the same period in Krebs solution alone (control). Date are shown as mean \pm SEM. Asterisk indicates $P<.05$ versus control group; hatch mark indicates $P<.05$ versus asymmetric dimethylarginine group; $\mathrm{n}=8,1$-way analysis of variance. $G A P D H$, Glyceraldehyde 3-phosphate dehydrogenase.

induce endothelial dysfunction by downregulating protein expression of eNOS and by increasing superoxide anion production and (2) these 2 mechanisms damaging the endothelial function may be partially reversed by the eNOS enhancer AVE3085. Because endothelial function is one of the major factors influencing the long-term patency of coronary artery bypass grafting conduits, these findings provide new insights in the protection of the endothelium of coronary bypass grafting conduits to improve the long-term patency of these grafts.

Nitric oxide has been widely regarded as the major mediator of endothelium-dependent vasodilation and plays a crucial role in the endothelium-mediated regulation of vascular homeostasis. It also regulates the vascular structure and cell-cell interactions in blood vessels. ${ }^{15}$ A relatively newly found and intriguing mechanism that leads to a reduction in nitric oxide is ADMA, an endogenous competitive inhibitor of eNOS that has been linked to endothelial dysfunction. ${ }^{2}$ Importantly, it has been demonstrated that elevated ADMA concentrations are found in patients with coronary artery disease and other related diseases, such as diabetes mellitus and chronic heart failure. ${ }^{4-6}$

It has been reported that ADMA induces the production of reactive oxygen species in endothelial cells, which may contribute to the senescence and apoptotic death of endothelial cells. It is now clear that ADMA affects superoxide anion production by uncoupling of eNOS and other eNOSindependent pathways. $^{2}$ In addition, superoxide anion production can attack the eNOS directly and induce

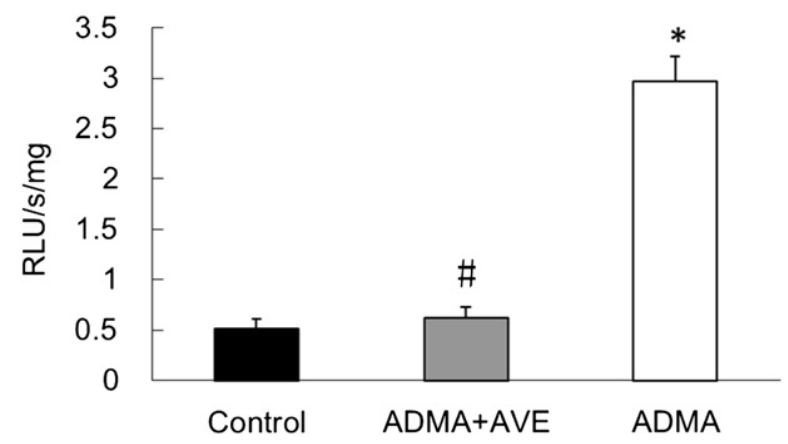

FIGURE 4. Superoxide production in human left internal thoracic artery rings. Vascular rings were incubated with asymmetric dimethylarginine (ADMA, $100 \mu \mathrm{mol} / \mathrm{L}$ ) in Krebs solution with or without the addition of AVE3085 (AVE, $30 \mu \mathrm{mol} / \mathrm{L})$ or in Krebs solution alone (control). Data are shown as mean \pm SEM. Asterisk indicates $P<.05$ versus control group; hatch mark indicates $P<.05$ versus asymmetric dimethylarginine group; $\mathrm{n}=7$; 1-way analysis of variance. $R L U$, Relative light units.

dissociation of eNOS dimer into monomer. ${ }^{16}$ One study has suggested that the decrease in nitric oxide production may be related to a reduction of eNOS expression by elevation of ADMA level in cultured endothelial cells. ${ }^{17}$

On the basis of these findings, the role of ADMA in the nitric oxide-related endothelial function of the human LITA vessels used as coronary artery bypass grafts is an important issue in the treatment of coronary artery disease. ADMA has been shown to be independently associated with maximum vasorelaxation in response to acetylcholine in saphenous veins, ${ }^{18}$ but whether ADMA affects the endotheliumdependent relaxation in human arteries is still unknown. In this study, we found that the acetylcholine-induced, endothelium-dependent relaxation was significantly reduced by incubation of the human LITA with ADMA. This finding clearly demonstrates that ADMA plays a role in reducing nitric oxide-mediated relaxation in human arteries.

As a novel small-molecular weight compound, AVE3085 increases endothelial nitric oxide production by the simultaneous upregulation of eNOS expression and reversal of eNOS uncoupling. ${ }^{7}$ Previous studies have indicated that long-term treatment with AVE3085 significantly increases eNOS messenger RNA and protein levels in the cardiac tissues of male Sprague-Dawley rats and male Dahl saltsensitive rats. ${ }^{89}$ Further, we have recently demonstrated that use of AVE3085 protects porcine coronary endothelium from ischemia-reperfusion injury and restores impaired endothelial function in a hypertensive model by significantly increasing the eNOS expressions of endothelial cells. ${ }^{10,11}$ In addition, AVE3085 has been shown to ameliorate endothelial dysfunction in $\mathrm{db} / \mathrm{db}$ mice through increased nitric oxide bioavailability, which reduces oxidative stress in the vascular wall. ${ }^{12}$

Interestingly, we found that the expression of eNOS was downregulated in human LITA specimens when they were 
incubated with AMDA and that the addition of AVE3085 significantly increased the eNOS protein level. These findings demonstrate that the reduction of the relaxation may be associated with the downregulation of eNOS, as Jiang and associates ${ }^{17}$ suggested that the decrease of the nitric oxide production may be related to reduction of eNOS expression by elevation of ADMA level in cultured endothelial cells.

It is well known that superoxide anion impairs vascular function. ${ }^{3}$ Recent studies showed that ADMA, an endogenous inhibitor of eNOS, uncoupled eNOS activity to generate superoxide anion by inhibiting heat shock protein 90 associated with eNOS. ${ }^{19}$ In accordance with other reports, we found in this study that ADMA could uncouple eNOS activity to increase superoxide anion generation in cultured internal thoracic arteries. In fact, we found that AVE3805 inhibited ADMA-induced superoxide anion generation. Our findings indicate that AVE3805 may be able to maintain the eNOS coupling activity to product nitric oxide in the human artery, which supports the potential use of AVE3805 to protect vascular endothelial function in human disease.

In this study, we tested the hypothesis that the newly developed eNOS enhancer AVE3085 could play a role in the restoration of the downregulated eNOS and therefore restore the acetylcholine-induced, endothelium-dependent relaxation to a certain extent. The results support this hypothesis, because incubation of LITA ring segments in AVE3085 (30 $\mu \mathrm{mol} / \mathrm{L}$ ) significantly improved the acetylcholineinduced relaxation that was reduced by ADMA alone. This study also demonstrated that such restoration of the endothelium-dependent relaxation is associated with eNOS and superoxide anion changes.

\section{Study Limitations}

This study is an in vitro experimental investigation at the tissue (vascular) and molecular levels. Any in vivo protective effect of AVE3085 on the endothelial function damaged by ADMA in the LITA and its potential clinical impact on the long-term patency of the graft remain to be defined.

\section{Clinical Implications}

Use of eNOS enhancers to restore the reduced nitric oxide-related endothelial function is a new therapeutic strategy that may have clinical implications in a number of clinical situations associated with endothelial dysfunction, such as diabetes, hypertension, and ischemia-reperfusion injury in coronary artery disease, cardiac surgery, or organ transplants. This study emphasizes that the use of eNOS enhancers may improve endothelial function in the human arteries used as grafts in coronary artery bypass grafting and thus supports the trial of those new drugs as a therapeutic strategy for the listed pathologic conditions.

\section{CONCLUSIONS}

In conclusion, the results of our study in the human LITA suggest that increased ADMA levels directly cause endothelial dysfunction by downregulating protein expression of eNOS and by increasing superoxide production. These mechanisms of endothelial dysfunction may be partially reversed by means of the eNOS enhancer AVE3085. These findings provide new insights into the protection of the endothelium of the coronary artery bypass grafting conduit to improve the long-term patency of the grafts.

The assistance of the surgical team (Dr Jing Wen-Bin) and nurses (En-Jie Yu and the team members) in the Cardiac Operating Theater, TEDA International Cardiovascular Hospital, is gratefully acknowledged.

\section{References}

1. Vallance P, Leone A, Calver A, Collier J, Moncada S. Accumulation of an endogenous inhibitor of nitric oxide synthesis in chronic renal failure. Lancet. 1992; 339:572-5

2. Jiang DJ, Jia SJ, Dai Z, Li YJ. Asymmetric dimethylarginine induces apoptosis via p38 MAPK/caspase-3-dependent signaling pathway in endothelial cells. J Mol Cell Cardiol. 2006;40:529-39.

3. Ou J, Ou Z, Jones DW, Holzhauer S, Hatoum OA, Ackerman AW, et al. An apolipoprotein A-1 mimetic, dramatically improves vasodilation in hypercholesterolemia and sickle cell disease. Circulation. 2003;107:2337-41.

4. Valkonen VP, Päivä H, Salonen JT, Lakka TA, Lehtimäki T, Laakso J, et al. Risk of acute coronary events and serum concentration of asymmetrical dimethylarginine. Lancet. 2001;358:2127-8.

5. Krzyzanowska K, Mittermayer F, Wolzt M, Schernthaner G. Asymmetric dimethylarginine predicts cardiovascular events in patients with type 2 diabetes. $\mathrm{Di}$ abetes Care. 2007;30:1834-9.

6. Dückelmann C, Mittermayer F, Haider DG, Altenberger J, Eichinger J, Wolzt M. Asymmetric dimethylarginine enhances cardiovascular risk prediction in patients with chronic heart failure. Arterioscler Thromb Vasc Biol. 2007;27:2037-42.

7. Wohlfart P, Xu H, Endlich A, Habermeier A, Closs EI, Hübschle T, et al. Antiatherosclerotic effects of small-molecular-weight compounds enhancing endothelial nitric-oxide synthase (eNOS) expression and preventing eNOS uncoupling. J Pharmacol Exp Ther. 2008;325:370-9.

8. Riad A, Westermann D, Van Linthout S, Mohr Z, Uyulmaz S, Becher PM, et al. Enhancement of endothelial nitric oxide synthase production reverses vascular dysfunction and inflammation in the hindlimbs of a rat model of diabetes. Diabetologia. 2008;51:2325-32.

9. Westermann D, Riad A, Richter U, Jäger S, Savvatis K, Schuchardt M, et al. Enhancement of the endothelial NO synthase attenuates experimental diastolic heart failure. Basic Res Cardiol. 2009;104:499-509.

10. Xue HM, He GW, Huang JH, Yang Q. New strategy of endothelial protection in cardiac surgery: use of enhancer of endothelial nitric oxide synthase. World $J$ Surg. 2010;34:1461-9.

11. Yang Q, Xue HM, Wong WT, Tian XY, Huang Y, Tsui SK, et al. AVE3085, an enhancer of endothelial nitric oxide synthase, restores endothelial function and reduces blood pressure in spontaneously hypertensive rats. $\mathrm{Br} J$ Pharmacol. 2011;16:1078-85.

12. Cheang WS, Wong WT, Tian XY, Yang Q, Lee HK, He GW, et al. Endothelial nitric oxide synthase enhancer reduces oxidative stress and restores endothelial function in db/db mice. Cardiovasc Res. 2011;92:267-75.

13. Bai XY, Liu XC, Jing WB, Yang Q, Tang XD, He GW. Effect of amlodipine in human internal mammary artery and clinical implications. Ann Thorac Surg. 2010;9:1952-7.

14. He GW, Liu ZG. Comparison of nitric oxide release and endothelium-derived hyperpolarizing factor-mediated hyperpolarization between human radial and internal mammary arteries. Circulation. 2001;104(12 Suppl. 1):I344-9.

15. Schmidt HH, Walter U. NO at work. Cell. 1994;78:919-25.

16. Zou MH, Shi C, Cohen RA. Oxidation of the zinc-thiolate complex and uncoupling of endothelial nitric oxide synthase by peroxynitrite. J Clin Invest. 2002; 109:817-26. 
17. Jiang JL, Zhang XH, Li NS, Rang WQ, Feng-Ye, Hu CP, et al. Probucol decreases asymmetrical dimethylarginine level by alternation of protein arginine methyltransferase I and dimethylarginine dimethylaminohydrolase activity. Cardiovasc Drugs Ther. 2006;20:281-94.

18. Antoniades C, Shirodaria C, Leeson P, Antonopoulos A, Warrick N, VanAssche $\mathrm{T}$, et al. Association of plasma asymmetrical dimethylarginine
(ADMA) with elevated vascular superoxide production and endothelial nitric oxide synthase uncoupling: implications for endothelial function in human atherosclerosis. Eur Heart J. 2009;30:1142-50.

19. Ou J, Ou Z, Ackerman AW, Oldham KT, Pritchard KA Jr. Inhibition of heat shock protein 90 (hsp90) in proliferating endothelial cells uncouples endothelial nitric oxide synthase activity. Free Radic Biol Med. 2003;34:269-76.

Access to The Journal of Thoracic and Cardiovascular Surgery Online is reserved for print subscribers!

Full-text access to The Journal of Thoracic and Cardiovascular Surgery Online is available for all print subscribers. To activate your individual online subscription, please visit The Journal of Thoracic and Cardiovascular Surgery Online, point your browser to http://www.mosby.com/itcvs, follow the prompts to activate your online access, and follow the instructions. To activate your account, you will need your subscriber account number, which you can find on your mailing label (note: the number of digits in your subscriber account number varies from 6 to 10). See the example below in which the subscriber account number has been circled:

\section{Sample mailing label}

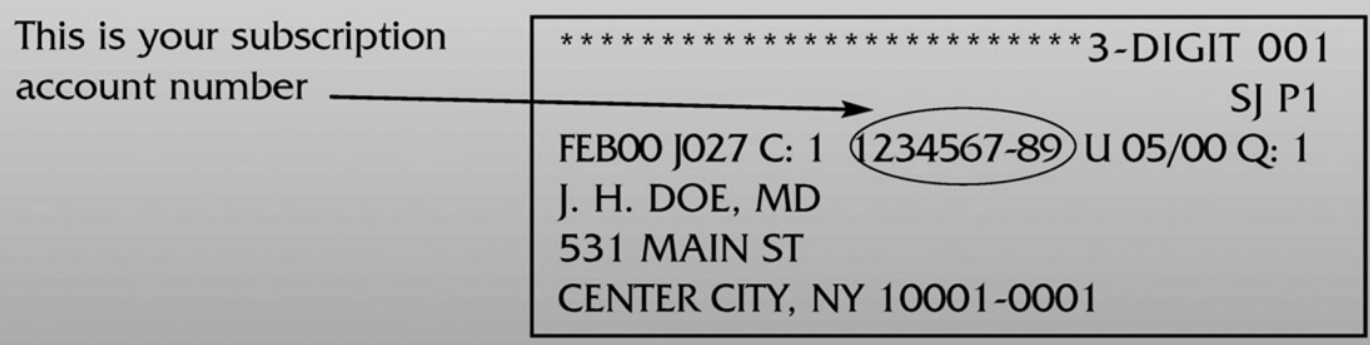

Personal subscriptions to The Journal of Thoracic and Cardiovascular Surgery Online are for individual use only and may not be transferred. Use of The Journal of Thoracic and Cardiovascular Surgery Online is subject to agreement to the terms and conditions as indicated online. 